\title{
ESSAI DE PHYTOCLIMATOLOGIE DYNAMIQUE DANS LE NORD DU PORTUGAL, ET REFLEXION SUR LES CLIMATS MEDITERRANEENS PORTUGAIS
}

\author{
ANA ISABEL D. CORREIA ${ }^{1}$ \\ MICHEL LECOMPTE ${ }^{2}$
}

\begin{abstract}
Résumé - La relation climat-végétation est étudiée d'une manière nouvelle dans le nord du Portugal, dans l'optique de la continuité dynamique du climat, susceptible de se traduire par l'existence de continuиms botaniques. Il a été procédé à un échantillonnage continu de la végétation au long d'un transect NW-SE de $185 \mathrm{~km}$, orienté selon le plus fort gradient udométrique. L'analyse floristique a allié une analyse multivariée à des applications de la théorie de l'information. L'étude du climat s'appuie principalement sur la description des champs spatiaux des précipitations quotidiennes durant trois années. Des analyses multivariées ont été également appliquées à ces descriptions, et à leur essai d'explication aérologique (Analyse Canonique des Correspondances). Un seuil de satisfaction hydrique, correspondant à environ $1400 \mathrm{~mm}$ de pluie annuelle moyenne, semble déterminer l'établissement, entre les Serras d'Alvão et Falperra et l'océan d'une végétation atlantique, floristiquement homogène, parce que les plantes seraient insensibles, passée cette valeur, aux flutuactions udométriques. Cette végétation se "défait" rapidement vers les régions orientales, plus sèches. La comparaison des régimes quotidiens de pluie entre les divers postes, et l'interprétation dynamique de leurs différences, éclairent la discussion sur l'appartenance atlantique ou méditerranéenne du climat du NW de la Péninsule Ibérique, où s'épanouit cette végétation atlantique. A vrai dire, la façade maritime occidentale du Portugal associe ces deux influences, et s'oppose ainsi aux provinces abritées de l'intérieur.
\end{abstract}

Mots-clés: Méditerranée, Portugal, Phytoclimatologie dynamique, Climatologie dynamique, continuит botanique, seuils biologiques.

\begin{abstract}
A study in Dynamic Phytoclimatology in the north of Portugal and the Question of Mediterranean climates in Portugal - The climate-vegetation relation in the north of Portugal is studied in a new way. The dynamic continuity of the climate is revealed by the existence of botanical continuиms. The vegetation sampling was made along a continuous NW-SE line transect, $185 \mathrm{~km}$ long, parallel to the strongest pluviometric gradient. Multivariate analysis and some of the applications of the information theory were used in the floristic study. In the climatic study the spatial patterns of daily values of precipitation were analysed for a period of three years. This description was also done by means of multivariate analysis, including CCA for the aerological explanation. A threshold of hydric satisfaction, around $1400 \mathrm{~mm}$ of mean annual rainfall seems to determine the existence of an Atlantic vegetation, between the Alvão and Falperra mountains and the Ocean. This vegetation is homogeneous because it does not respond to the udometric variations above this level. To the east, the Atlantic vegetation rapidly dissapears. The comparison between

\footnotetext{
${ }^{1}$ Professora Auxiliar do Depart. Biologia Vegetal, Faculdade de Ciências da Universidade de Lisboa, Campo Grande, Bloco C2,.4 Piso, P 1700 Lisboa. Investigadora do Centro de Biologia Ambiental, FCUL.

2 Professor na Universidade de Paris 7, Laboratoire de Géographie Physique, Université Paris 7 Denis Diderot, 2 place Jussieu, F 75 005, Paris.
} 
daily precipitation regimes in the different stations and the dynamic interpretation of the differences brings some light to the discussion about the climate in the NW of the Iberian Peninsula: is it an Atlantic or a Mediterranean climate? In the maritime coast of Portugal these two influences mingle, as opposed to the sheltered interior provinces.

Key-words: Mediterranean area, Portugal, dynamic Phytoclimatology, dynamic Climatology, botanical continuum, biological thresholds.

Resumo - Ensaio de fitoclimatologia dinâmica no Norte de Portugal e reflexão sobre os CLIMAS MEDitERRÂNeOS PORTUGUESES - A relação clima-vegetação é estudada de forma inovadora no Norte de Portugal. Este estudo é realizado na óptica da continuidade dinâmica do clima, susceptível de se traduzir botanicamente na existência de um continuum. A amostragem da vegetação foi orientada de modo a acompanhar o mais forte gradiente udométrico, seguindo um transecto NW-SE com $185 \mathrm{~km}$ de comprimento. No estudo florístico utilizaram-se as análises multivariadas e algumas das aplicações da teoria da informação. O estudo climático baseou-se essencialmente na descrição dos campos espaciais das precipitações diárias em três anos consecutivos. As análises multivariadas também foram usadas nesta descrição e procurou-se a sua explicação aerológica através da Análise Canónica das Correspondências. Um limiar de satisfação hídrica, correspondente a cerca de $1400 \mathrm{~mm}$ de precipitação média anual, parece permitir a existência de uma vegetação atlântica entre as Serras de Alvão e Falperra e o Oceano. Esta vegetação é homogénea, já que se revela insensível às variações de precipitação acima daquele valor, e "desfaz-se" rapidamente nas regiões orientais, mais secas. A comparação dos regimes diários de precipitação entre os vários postos e a interpretação dinâmica das suas diferenças relança a discussão sobre o carácter, atlântico ou mediterrânico, do clima do NW da Península Ibérica. A fachada marítima occidental de Portugal associa estas duas influências, opondo-se ao interior, mais abrigado.

Palavras chave: zona mediterrânica, Portugal, Fitoclimatologia dinâmica, Climatologia dinâmica, continuum botânico, limiar biológico.

\section{1 - INTRODUCTION ET RAPPEL TECHNIQUE}

La phytoclimatologie dynamique (LECOMPTE, 1987, 1988, 1989) se présente comme une alternative aux approches phytogéographiques régionales classiques. Celles-ci n'utilisent généralement qu'un nombre réduit de références climatiques, essentiellement des valeurs moyennes de précipitation et de température. Ces valeurs, obtenues en quelques points des réseaux climatologiques, laissent de côté la plus grande partie de la variabilité spatio-temporelle du climat, qu'entraîne naturellement la dynamique changeante de l'atmosphère.

Cette appréhension moyenne et "ponctiforme" du climat s'accorde à la vision traditionnelle de la phytogéographie européenne, tournée vers la recherche d'ensembles phytoclimatiques homogènes, juxtaposés en structures discontinues (EMBERGER, 1930; GUINOCHET, 1973; AlBUQUERQUE, 1954; RIVAS-MARTINEZ, 1982, etc...): la référence à quelques observations climatiques dispersées est bien faite pour attester des discontinuités supposées ! La réalité de celles-ci est pourtant douteuse dans les champs continus et ô combien fluctuants du climat, où l'homogénéité phytoclimatique ne semble pouvoir, en conséquence, être la règle (LECOMPTE et ALEXANDRE, 1996).

La phytoclimatologie dynamique est née de la constatation que gradients climatiques et continuums botaniques (WHITTAKER, 1967), dont 
l'orientation peut être perçue à travers la géographie des paysages (HuMBOLDT, 1807) ou des cartes de valeurs climatiques moyennes, sont liés entre eux, et que leurs variations peuvent être analysées conjointement (LECOMPTE, 1973, 1981, 1986).

Cette analyse peut porter sur les distributions des espèces et la circulation de l'atmosphère le long des gradients climatiques. On s'attend en effet à ce que les espèces végétales se succèdent dans l'espace en suivant de près les directions et les rythmes imposés aux variations du climat par les phénomènes dynamiques. Les transitions climatiques, auxquelles correspondent les modifications de la flore les plus rapides, peuvent être interprétées comme les limites d'influence de circulations atmosphériques contrastées.

Les traits généraux de la distribution linéaire des espèces sont fixés par l'Analyse Factorielle des Correspondances (AFC), dont les premiers axes sont assimilables à des variables floristiques synthétiques, exprimant chacune l'un de ces traits; le détail de ceux-ci peut être précisé par des analyses de fréquence de chaque espèce, dans l'optique de la théorie de l'information (GODRON, 1967 et 1971). Ces diverses statistiques sont appliquées à des échantillons de relevés segmentaires de longueur constante, qui se succèdent le long de transects orientés comme les gradients climatiques dominants. Les détails de la procédure sont donnés par LECOMPTE $(1973,1986)$.

L'étude de la variabilité spatio-temporelle du climat s'appuie quant à elle sur la description de champs quotidiens de précipitation et température, éclairée par les circulations atmosphériques qui les génèrent. Les circulations atmosphériques sont décrites d'après les bulletins météorologiques quotidiens, comme le Bulletin Météorologique Européen (BME), et classées selon des typologies inspirées de DELANNOY et LECOMPTE (1975) et RONCHAIL et LECOMPTE (1981); y sont distingués la circulation générale en altitude $(500 \mathrm{hP})$ et au sol, et le flux régional, à un ou deux moments de la journée.

Les structures spatiales des champs quotidiens, et les structures temporelles des régimes stationnels sont déduites d'analyses factorielles portant sur des ensembles de centaines de journées de précipitation et de température maximale et minimale (DELANNOY et LECOMPTE, 1980, DELANNOY et al., 1979). Ces structures sont expliquées par certains caractères de la circulation atmosphérique. L'Analyse Canonique des Correspondances (ACC), qui intègre un modèle de régression multiple aux classiques analyses factorielles permet d'objectiver les explications apportées.

L'Analyse Canonique des Correspondances, ou Analyse Factorielle sur Variables Instrumentales a été conçue par TER BRAAK (1986) et LEBRETON et al. (1988a et 1988b) comme la recherche d'une combinaison linéaire de variables explicatives, qualitatives ou quantitatives, maximisant la variance de variables conditionnelles dépendantes. L'ACC équivaut à l'AFC d'un nuage de descripteurs (i.e. les pluies quotidiennes) reconstitué par régression multiple sur un ensemble de variables explicatives indépendantes, ainsi recombinées. Elle aboutit, après une double rotation 
d'axes, à la définition de variables explicatives synthétiques, "variables canoniques", superposables aux facteurs principaux de l'AFC des descripteurs réels qu'elles sont supposées expliquer. Dans le cas des variables qualitatives, cette explication est facilitée par le calcul des corrélations entre classes des variables explicatives de départ et variables canoniques, que l'on représente sous forme de cercles de corrélation (fig. 5). La pertinence de l'explication globalement apportée s'apprécie par la conformité du nuage reconstitué des descripteurs à celui des descripteurs réellement observés ou mesurés. On trouvera les détails techniques dans les deux publications de LEBRETON et al., et l'application informatique dans le logiciel BIOMECO du CNRS.

\section{2 -PRÉSENTATION DU TRANSECT FLORISTIQUE ET DE SON ENVIRONNE- MENT CLIMATIQUE}

Cette nouvelle approche phytoclimatique est appliquée au nord du Portugal (CORREIA, 1994), où les gradients de précipitation sont particulièrement remarquables: de 400 à plus de 3000 $\mathrm{mm}$ de pluie entre Espagne et Atlantique. L'uniformité des roches, essentiellement granitiques, a simplifié l'interprétation de la relation climat-végétation; de légers décalages du cheminement botanique ont été consentis pour éviter de traverser les affleurements de schistes.

L'étude floristique est conduite au long d'un transect de $185 \mathrm{~km}$, prenant en écharpe le territoire du NW vers le SE, en enchaînant 295 relevés segmentaires de 500m environ chacun (fig. 1); la chaîne des relevés est interrompue au niveau des terroirs cultivés. Du côté de l'Atlantique, le transect commence dans la basse vallée du Minho, puis il escalade les plus hauts reliefs du NW (serras de Peneda, Gerês, Cabreira et Alvão), séparés les uns des autres par des vallées souvent profondes. Sont traversés ensuite les plateaux de Trás-os-Montes, de l'Alto Douro et de la Beira intérieure, incisés par les vallées du Douro et de ses affluents, notamment le Tua et le Côa (fig. 2).

L'orientation du transect a été choisie selon le plus fort gradient udométrique sur la carte des isohyètes moyennes annuelles de DAVEAU (1977). La géographie des paysages botaniques, qui visualise parfois remarquablement l'orientation des gradients climatiques, s'est avérée ici peu utile, du fait de la profonde désorganisation historique de ces paysages. Pourtant, ce désordre apparent n'a pas fait disparaître toute relation entre la distribution de la flore et le climat, comme on pourra le voir. D'ailleurs, les variations floristiques analysées se révèleront, dans une vision a posteriori, coïncider avec des changements de physionomie des paysages, discrets, mais nets!

L'environnement climatique du transect floristique est précisé à partir d'une sélection de trente-quatre postes udométriques ou stations, qui l'enveloppe par une bande d'une vingtaine de kilomètres de large (fig. 1). Les moyennes en sont disponibles pour des durées variables. Par ailleurs, le réseau de ces postes a fourni une approche des champs thermiques et de précipitation quotidiens; ceux-ci étant reliés ensuite à la circulation atmosphérique sur le nord du Portugal (tab. 1, types et fréquences). La distribution géographique des pluies est de loin la plus contrastée, et c'est 
donc elle qui retient ici l'attention. 749 journées pluvieuses des années 1987 à 1989 permettent de préciser ces contrastes, que l'on rapproche de la distribution de la flore. Une journée est qualifiée de pluvieuse lorsque l'un des postes a reçu au moins $1 \mathrm{~mm}$ de pluie.

\section{Légende de la figure 1 (page suivante)}

Monção/Valinha
Lamas de Mouro
Parada (Monção)
Fonte Boa
Padrão
Bouça dos Homens
Extremo
Senhora da Peneda
Cabana Maior
Soajo
Lindoso
Leonte
Covide

$\begin{array}{ll}14 & \text { Paradela do Rio } \\ 15 & \text { Xertelo } \\ 16 & \text { Pedra Bela/Gerês } \\ 17 & \text { Salamonde } \\ 18 & \text { Zebral } \\ 19 & \text { Venda Nova } \\ & \\ 20 & \text { Couto } \\ & \text { Dornelas } \\ 21 & \text { Vidago } \\ 22 & \text { Salto } \\ 23 & \text { Ribeira de Pena } \\ 24 & \text { Cavês } \\ 25 & \text { Lixa do Alvão } \\ 26 & \text { Lamas de Alvadia }\end{array}$

Minas de Jales
Lamas de Olo
Torre do Pinhão
Vila Chã
Folgares
Fontelonga
Moncorvo
Freixo de Numão
Castelo Melhor
Escalhão
Vale de Afonsinhos
Fig. Cast. Rodrigo
Tibo da Gavieira

$\begin{array}{ll}40 & \text { Montalegre } \\ 41 & \text { Ponte da Barca } \\ 42 & \text { Ermida/Gerês } \\ 43 & \text { Guilhofrei/Barragem } \\ 44 & \text { Pedras Salgadas } \\ 45 & \text { Cabeceiras de } \\ & \text { Baixo } \\ 46 & \text { Vila Real } \\ & \\ 47 & \text { Régua } \\ 48 & \text { Pinhão/Sta Bárbara } \\ 49 & \text { Vesúvio } \\ 50 & \text { Muxagata } \\ 51 & \text { Almendra } \\ 52 & \text { Vermiosa }\end{array}$




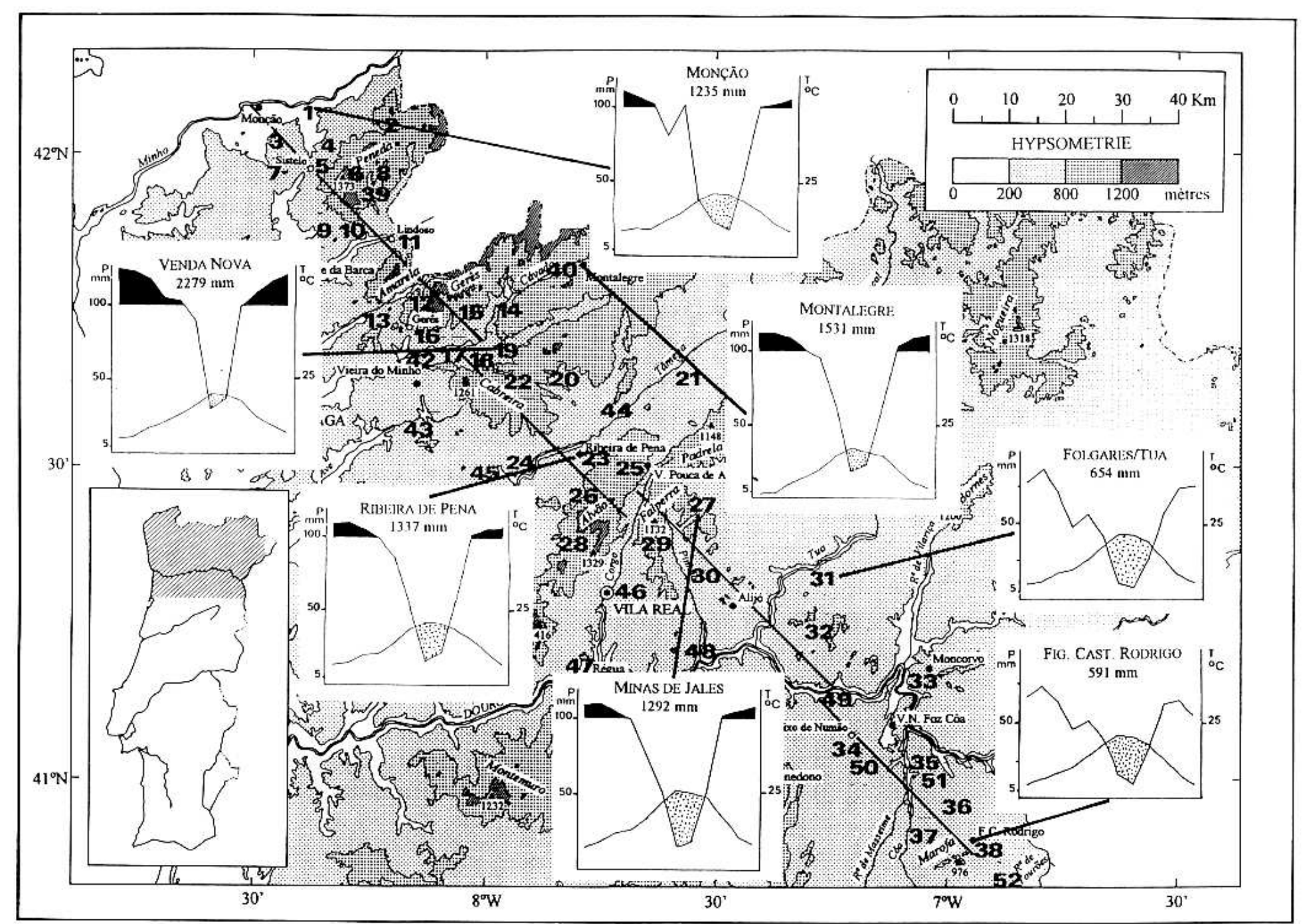

Figure 1 - Carte de localisation du transect, des stations météorologiques et postes udométriques; pour les stations sélectionnées sont indiqués le total de précipitation et le diagramme ombrothermique (adapté de CoRREIA, 1994).

Figura 1 - Localização do transecto, das estações meteorológicas e dos postos udométricos. 
Tableau I - Fréquences absolues et relatives des différents types de circulation en altitude et au sol à 0 heure pour les journées pluvieuses des années 1987, 88 et 89, par saison et pour le total des 749 journées pluvieuses (dernière colonne)

Quadro II - Frequências absolutas e relativas dos diferentes tipos de circulação em altitude e junto ao solo às 0 horas para os dias chuvosos dos anos 1987, 88 e 89, por estação e para o total dos 749 dias chuvosos (última coluna)

\begin{tabular}{|c|c|c|c|c|c|c|c|c|c|c|}
\hline \multirow[b]{2}{*}{ Situation en altitude } & \multicolumn{2}{|c|}{ hiver } & \multicolumn{2}{|c|}{ printemps } & \multicolumn{2}{|c|}{ été } & \multicolumn{2}{|c|}{ automne } & \multicolumn{2}{|c|}{ Année } \\
\hline & tot & $\%$ & & $\%$ & & $\%$ & & $\%$ & tot: & $\%$ \\
\hline Zonale & 91 & 47 & 99 & 44 & 42 & 34 & 64 & 32 & 296 & 40 \\
\hline Dorsale anticyclonique & 42 & 22 & 24 & 10 & 12 & 10 & 35 & 17 & 113 & 15 \\
\hline Axe de vallée & 18 & 9 & 23 & 10 & 20 & 16 & 23 & 11 & 84 & 11 \\
\hline Versant $\mathrm{W}$ de vallée & 7 & 4 & 20 & 9 & 5 & 4 & 8 & 4 & 40 & 5 \\
\hline Versant E de vallée & 14 & 7 & 15 & 7 & 12 & 10 & 50 & 24 & 91 & 12 \\
\hline $\begin{array}{l}\text { Dépression en altitude et/ou } \\
\text { goutte froide }\end{array}$ & 18 & 9 & 42 & 18 & 31 & 24 & 19 & 9 & 110 & 15 \\
\hline Marais barométrique & 3 & 2 & 4 & 2 & 3 & 2 & 5 & 3 & 15 & 2 \\
\hline
\end{tabular}

\begin{tabular}{|c|c|c|c|c|c|c|c|c|c|c|}
\hline Situation au sol & & & & & & & & & & \\
\hline $\begin{array}{l}\text { Anticyclonique méridienne de } \\
\mathrm{N} \text {, de NW ou de NE }\end{array}$ & 7 & 4 & 19 & 8 & 32 & 25 & 14 & 7 & 72 & 10 \\
\hline $\begin{array}{l}\text { Anticyclonique méridienne de } \\
\text { S ou de SE }\end{array}$ & 11 & 6 & 2 & 1 & 0 & 0 & 5 & 2 & 18 & 2 \\
\hline Anticyclonique - flux d' W & 28 & 14 & 36 & 16 & 44 & 35 & 19 & 9 & 127 & 17 \\
\hline Anticyclonique - flux d' E & 20 & 10 & 14 & 6 & 8 & 7 & 16 & 8 & 58 & 8 \\
\hline $\begin{array}{l}\text { Dépressionnaire méridienne de } \\
\mathrm{NW}, \mathrm{N} \text { ou NE }\end{array}$ & 6 & 3 & 47 & 21 & 12 & 10 & 16 & 8 & 81 & 11 \\
\hline $\begin{array}{l}\text { Dépressionnaire méridienne de } \\
\text { SW, de } \mathrm{S} \text { ou de SE }\end{array}$ & 13 & 7 & 7 & 3 & 0 & 0 & 40 & 20 & 60 & 8 \\
\hline Dépressionnaire zonale d' W & 93 & 48 & 83 & 36 & 10 & 8 & 81 & 40 & 267 & 35 \\
\hline $\begin{array}{l}\text { Dépression centrée sur la P. } \\
\text { Ibérique }\end{array}$ & 6 & 3 & 13 & 6 & 13 & 10 & 10 & 5 & 42 & 6 \\
\hline Marais barométrique & 9 & 5 & 6 & 3 & 6 & 5 & 3 & 1 & 24 & 3 \\
\hline $\begin{array}{l}\text { Total des circulations anticy- } \\
\text { cloniques }\end{array}$ & 56 & 34 & 71 & 31 & 84 & 67 & 54 & 26 & 275 & 37 \\
\hline $\begin{array}{l}\text { Total des circulations dépres- } \\
\text {-sionnaires }\end{array}$ & 118 & 61 & 150 & 66 & 35 & 28 & 147 & 73 & 450 & 60 \\
\hline
\end{tabular}




\section{3 - RÉSULTATS}

\section{1 - Une remarquable transition floristique à l'Est du Tua}

Le tableau des présences de 199 espèces pérennes est soumis à l'analyse factorielle des correspondances. Sur la figure 2, les variations des coordonnées des 295 relevés, ordonnés en ligne, relativement aux trois premiers axes de l'AFC, dessinent trois courbes brisées. Celles-ci indiquent les traits essentiels de la distribution linéaire de la flore échantillonnée.

La courbe correspondant au premier axe floristique (7\% de la variance totale) oppose nettement les deux tiers occidentaux du transect au tiers oriental. Les coordonnées constamment positives, ou presque, des relevés de l'ouest dénotent l'individualité d'une flore "atlantique", dont l'uniformité contraste avec la diversité de la flore "continentale", à l'est de la vallée du Tua; la mise en place de celle-ci se traduit par la décroissance rapide de la courbe, précédée par un court palier au niveau du plateau du Freixo. Dans une vision chorologique plus globale, l'importance biogéographique de ce secteur est évoquée par DUPONT (1962).

Les profondes vallées entre Atlantique et Tua rompent toutefois l'uniformité de la flore atlantique, comme le montre une série d'inflexions de la courbe de ce premier facteur. Celles-ci s'expliquent par l'installation d'espèces xériques à l'abri de ces vallées, à l'exemple de celle du Tua: la chute brutale des coordonnées y coïncide précisément avec l'adret de la vallée, soudainement enrichi en espèces d'affinités orientales (fig. 3), dont une partie disparaît sur l'ubac, en provoquant une remontée momentanée de la courbe. Contrairement à ces vallées occidentales, celles de l'est (Douro), ne semblent pas avoir d'influence sur la variation de la flore.

Le deuxième axe floristique (4\% de la variance) singularise les relevés d'altitude des serras occidentales. Le troisième axe $(3,5 \%)$ distingue les ultimes relevés à l'est du transect, où les schistes remplacent les granites.

L'analyse factorielle distingue par conséquent, sur les trois premiers facteurs, trois fractions nettement structurées de la flore, dont la contribution à la variance totale est néanmoins modeste, moins de $15 \%$. Cette valeur est sensiblement inférieure à celles relevées par LECOMPTE (1986) dans le Moyen-Atlas central marocain, ou par ALEXANDRE (1996) et GENIN (1997) en France méridionale, de l'ordre de 20 à 25\%. Elle est comparable en revanche aux chiffres de DERRAZ (1993) et EL AlAMI (1993), respectivement dans le Moyen-Atlas septentrional et le Rif. Cette modestie est attribuable à la désorganisation de la végétation par l'homme, dont la banalisation de nombreuses espèces est le résultat habituel. 


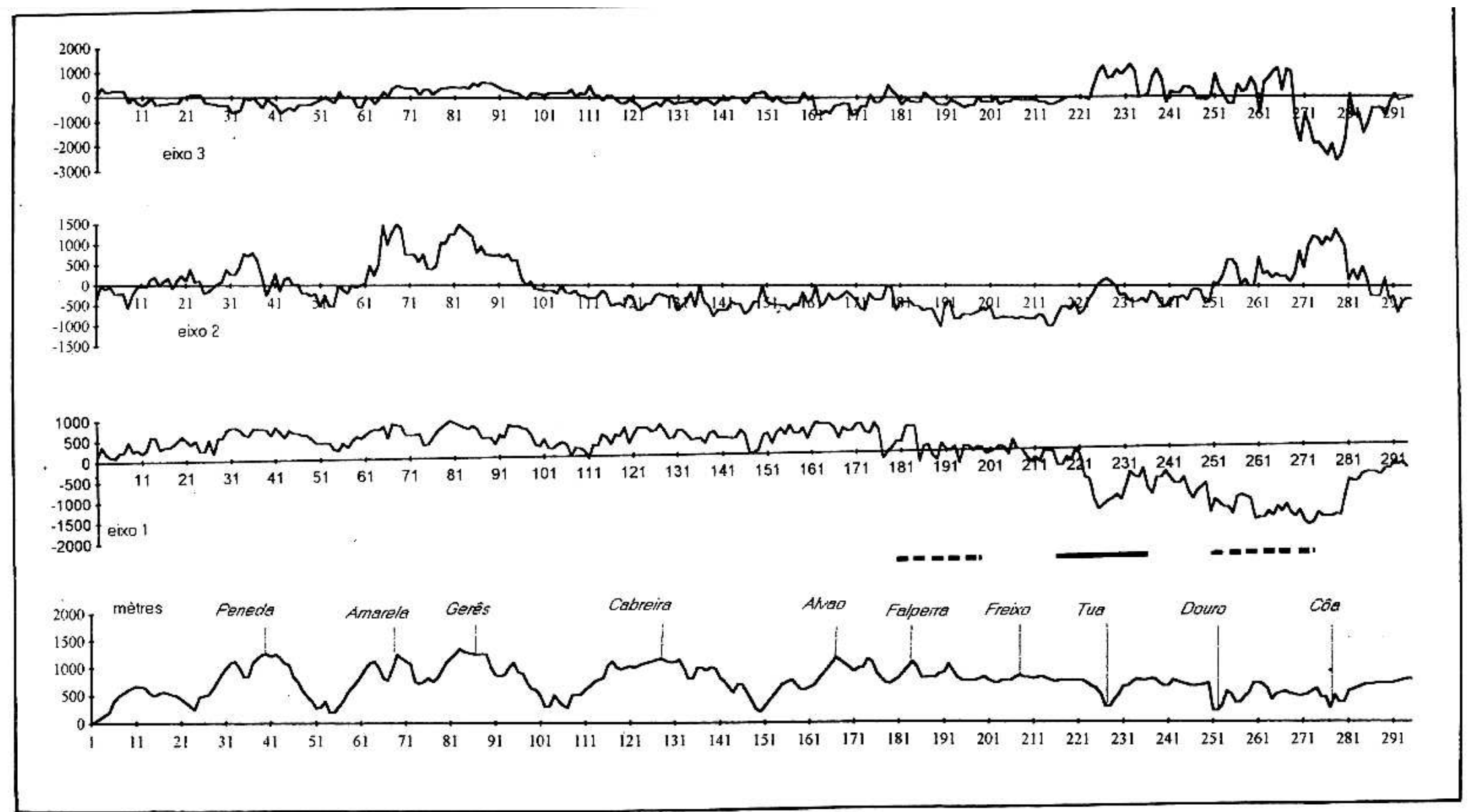

Figure 2 - Variations des trois premiers facteurs de l'analyse factorielle de la flore vivace le long du transect, avec l' indication des lieux où les sommes d' information fournies par les débuts et les fins des espèces sont particulièrent significatives (CORREIA, 1994).

Figura 2 - Variação dos três primeiros factores da AFC da flora vivaz ao longo do transecto, e locais em que as somas de informação dadas pelos inícios e pelos fins das espécies são particularmente significativas. 
La figure 3 représente la distribution des espèces, selon leur ordre d'apparition d'ouest en est, parmi l'ensemble des relevés. L'enveloppe du tableau des présences est équivalente à une classique "courbe aire-espèces", dont la forme confirme la dualité floristique du transect, révélée par l'AFC. La convexité de cette courbe dans sa partie W traduit l'homogénéité d'ensemble de la flore occidentale. Son redressement marqué, vers l'est, résultat d'un afflux d'espèces nouvelles, témoigne au contraire du caractère de transition de la flore correspondante. Un petit cortège d'altitude s'individualise entre les relevés 30 et 100 précédemment distingués par le deuxième axe de l'AFC, et quelques espèces liées aux relevés sur schistes, distingués par l'axe 3 , apparaissent tout au bas de la courbe.

Le calcul des probabilités permet de s'assurer de la signification statistique de divers attributs des distributions des présences des espèces (GODRON, 1967, 1971, GAUTHIER et al., 1977): par exemple, de leur début et de leur fin sur le transect, compte-tenu de leur fréquence. La théorie de l'information permet en outre, en additionnant des expressions logarithmiques de ces probabilités pour une série de relevés adjacents, d'apprécier la réelle importance des inflexions de la courbe aire-espèces. Ces calculs statistiques renforcent l'objectivité des comparaisons entre les informations floristiques qui s'inscrivent sur cette courbe.

Ainsi, la séquence des relevés de 218 à 227 concentre-t-elle la plus grande quantité d'information liée aux débuts et aux fins d'espèces sur l'ensemble de la ligne (270 bits). L'apparition de Thymus mastichina, Halimium viscosum, Cistus salvifolius, Juniperus oxycedrus, Rumex induratus, Erysimum linifolium, Phillyrea angustifolia, Pistacia terebinthus, Aristolochia longa, Psoralea bituminosa, Urginea maritima, Phagnalon saxatile, Armeria duriensis, Quercus faginea, Cistus ladanifer... et la disparition d'Ulex minor et d'Halimium alyssoides sont les plus significatives de cette évolution majeure de la végétation au niveau du Tua. D'autres traits de la structure floristique du transect sont soulignés, comme l'individualité de la flore montagnarde, ou du cortège des schistes... ou encore, la régression esquissée de la flore atlantique sur le plateau du Freixo, avec, notamment, la disparition significative de Quercus robur (fig. 2 et 3 ).

Figure 3 - Distribution des espèces le long du transect: en abcisses, les relevés, en ordonnées, et du haut vers le bas, les espèces par ordre d'apparition sur le transect, du NW vers le SE. Seules sont représentées les espèces apportant une information liée aux débuts et aux fins $\geq 20$ bits. (CORREIA, 1994).

Figura 3 - Distribuição das espécies ao longo do transecto. Só se representaram as espécies com uma quantidade de informação ligada ao seu início ou ao seu fim $\geq 20$ bits. 


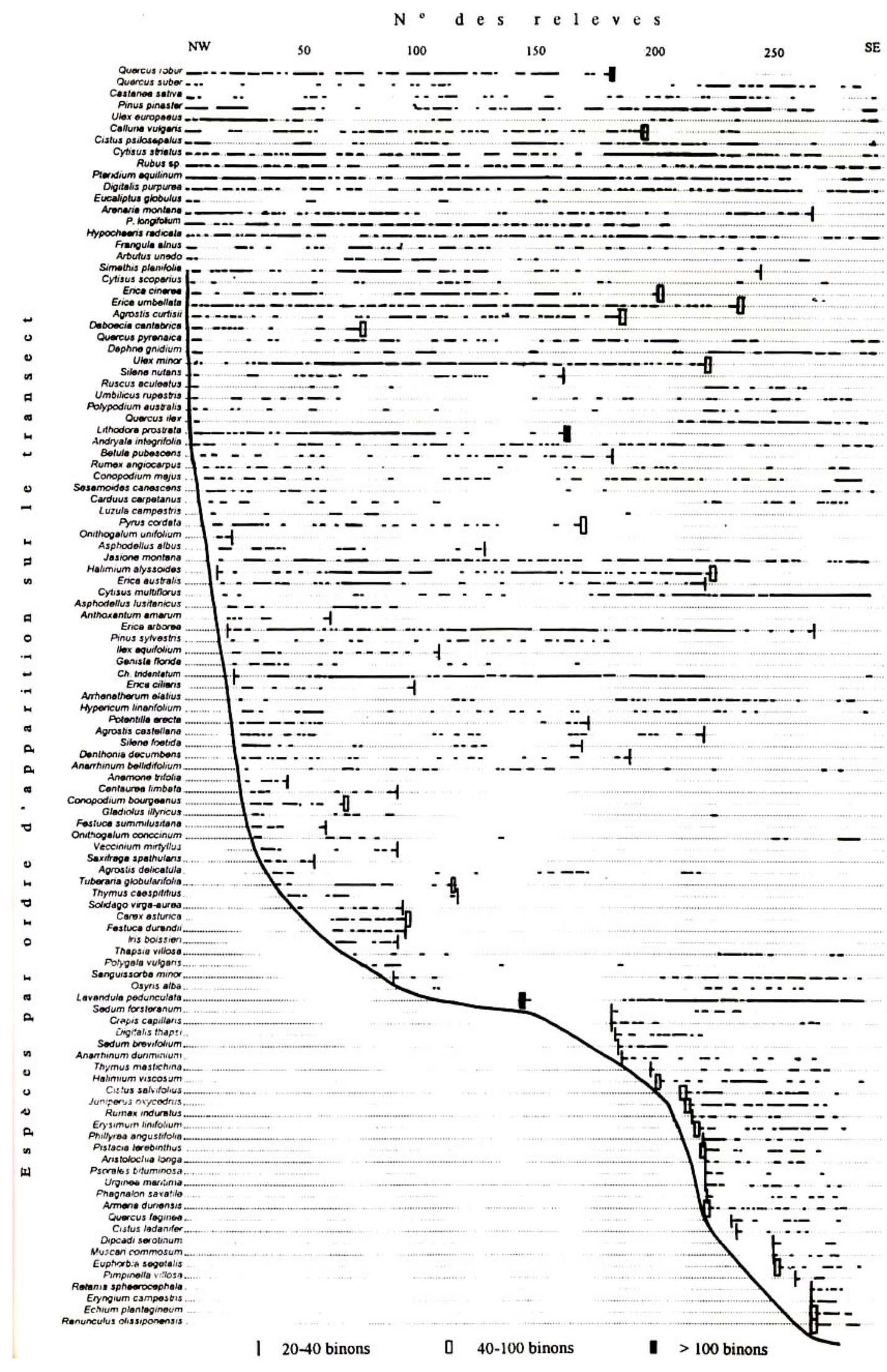




\section{2 - Le gradient climatique}

La comparaison des valeurs moyennes de précipitation et de température fait ressortir l'opposition des extrêmes géographiques est et ouest, reflétée par la végétation. Des analyses "d'information-mutuelle" entre les distributions des espèces et des diverses variables de l'environnement biophysique attestent de l'influence dominante de celles dont dépend le climat (éloignement de la mer, altitude....) sur la modification de la flore. Les modalités de cette influence, sa concomitance avec les changements les plus significatifs de la flore restent cependant à préciser.

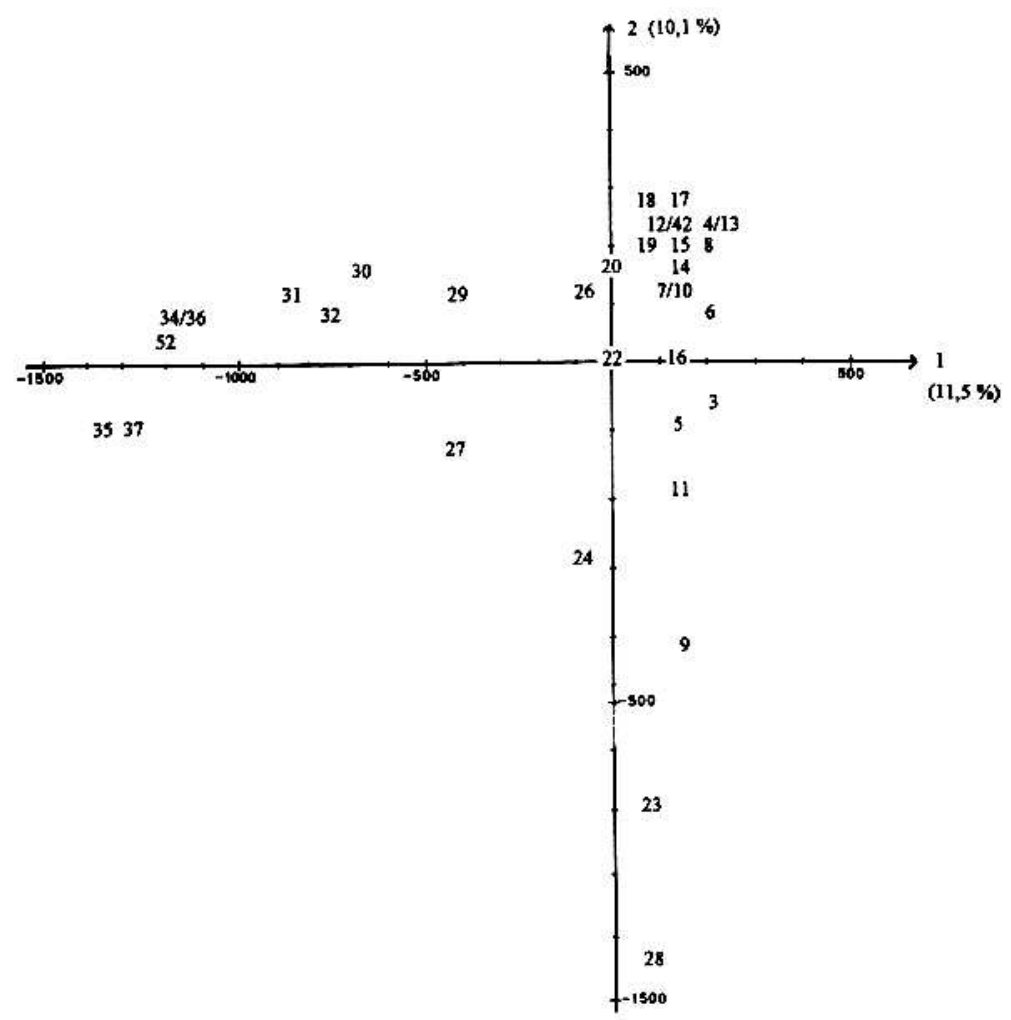

Figure 4 - Régimes pluviométriques quotidiens des 34 postes, ou stations, sur le plan 1-2 de l'AFC des précipitations quotidiennes, pour 749 journées pluvieuses des années 1987 à 1989 (au moins 1mm de pluie sur l'un des 34 postes ou stations). Les nombres renvoient à la figure 1. Les coordonnées des postes ou stations occidentaux sont très proches sur l'axe 1, le seul interprétable par l'ACC, et elles s'égrennent au contraire le long de cet axe vers l'est: uniformité des régimes en-deçà de la serra de Falperra, diversité au-delà. (CORREIA, 1994).

Figura 4 - Regimes pluviométricos diários dos 34 postos ou estações no plano factorial 1-2 da AFC das precipitações diárias para os 749 dias chuvosos dos anos 1987 a 1989 (pelo menos 1mm de chuva num dos 34 postos ou estações). Os números remetem para a figura 1. As coordenadas dos postos ou estações ocidentais estão muito próximos no eixo 1, o único interpretável da ACC, et distribuem-se ao longo dele: uniformidade de regimes a Oeste da serra da Falperra e diversidade para Este. 
La synthèse factorielle, par l'AFC, de la variabilité des champs quotidiens de précipitation, permet d'affiner la géographie climatique sous-jacente à celle de la végétation. La figure 4 montre la grande similitude de la distribution quotidienne des précipitations des postes occidentaux, dont les projections sur le plan 1-2 sont bien groupées. Ceux-ci répondent donc avec ensemble aux dynamiques pluvieuses, si l'on ne tient pas compte des montants absolus des abats, qui dépendent principalement de l'altitude. La situation s'inverse à l'est de la serra de Falperra, où prévaut la diversité des régimes quotidiens.

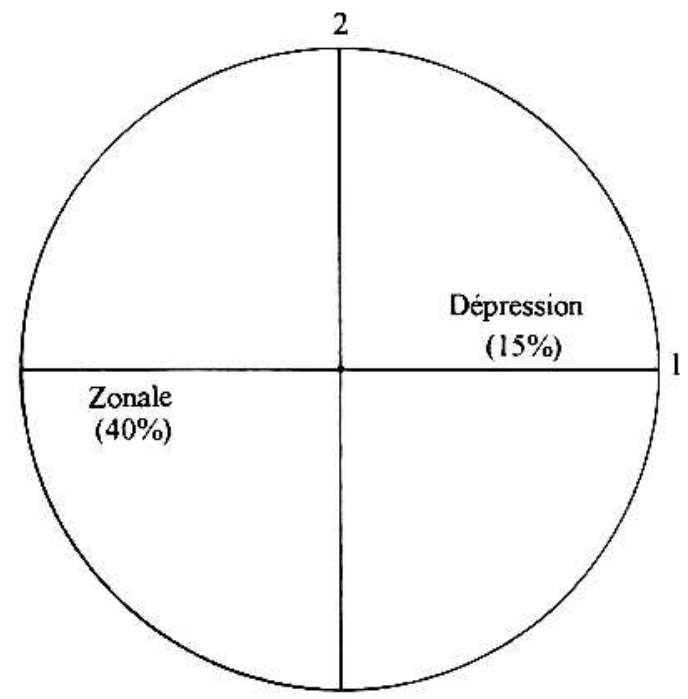

(a) En altitude

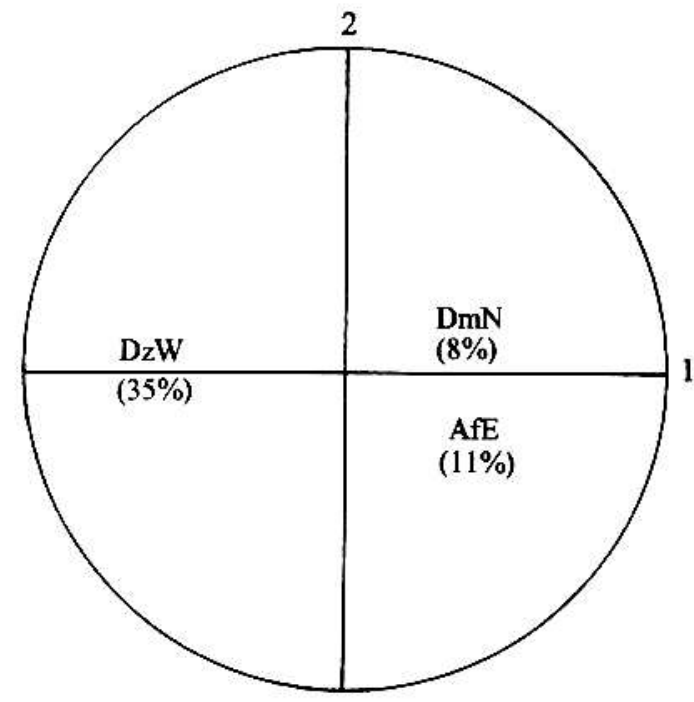

(b) Au sol à 0 heures

AfE - Anticyclonique flux d'E

DmN - Dépressionaire méridienne de NW, N ou NE

DzW - Dépressionaire zonale de W

Figure 5 - Analyse Canonique des Correspondances relative aux variables aérologiques. Les corrélations de celles-ci avec les deux premiers axes factoriels de l'AFC des précipitations quotidiennes sont d'autant plus fortes que leurs projections sur le plan sont proches de l' axe et de la circonférence, qui correspond à la corrélation 1. Seul l'axe 1, qui exprime l'opposition des régimes quotidiens de pluie est et ouest, est bien corrélé à certaines situations (corrélations >0.35): à deux types de circulation générale en altitude (a) et à trois types de situations au sol (b) (CORREIA, 1994). (entre parenthèses, le pourcentage d'occurence des différentes situations).

Figura 5 - Análise Canónica das Correspondências relativa às variáveis aerológicas. As correlações destas com os dois primeiros eixos factoriais da $\mathrm{AFC}$ das precipitações diárias são tanto mais fortes quanto mais próximas do eixo e da circunferência (que corresponde à correlação 1) estiverem as suas projecções no plano. Só o eixo 1, que exprime a oposição dos regimes diários de chuva oriental e ocidental, está bem correlacionado com certas situações (correlações > 0.35) (a dois tipos de situação em altitude (a); a três tipos à superfície (b). 
Quels sont les fondements aérologiques de ces observations? L'application par analyse canonique des types de circulation atmosphérique à l'AFC des champs de pluie quotidiens fournit une explication du premier axe de cette analyse factorielle. Les classes de circulation les mieux corrélées avec cet axe sont représentées sur les cercles de corrélations de la figure 5, séparément pour la circulation en altitude, et pour la circulation générale au sol à 0 heure. La pertinence de l'ACC apparaît en effet meilleure à cette heure.

En altitude, ce sont des circulations zonales qui sont responsables des journées de pluies particulièrement fortes sur la moitié occidentale du transect, et des dépressions et gouttes froides sur la moitié orientale.

$\mathrm{Au}$ sol, des situations zonales d'ouest sont liées aux plus fortes précipitations sur l'ouest, et des circulations dépressionaires méridiennes de secteur $\mathrm{N}$ et des marges d'anticyclones d'E, à celles intéressant principalement l'est.

L'analyse canonique manifeste donc clairement l'influence de certaines circulations pluvieuses sur la discrimination de la distribution quotidienne des précipitations de l'ouest et de l'est du pays, la transition entre ces régimes, vers le milieu du transect, pouvant être interprétée comme une limite entre influences contraires (MOUNIER, 1979). La dynamique frontale des perturbations polaires explique le comportement homogène des postes du Portugal atlantique, balayés par les mêmes fronts, souvent générateurs de fortes précipitations. Au contraire, dans le Portugal intérieur et en Espagne proche, l'instabilité verticale, provocant la pluie tantôt dans un lieu, tantôt dans un autre, produit autant de types de distribution des pluies quotidiennes qu'il y a de localités; ces types se rapprochent petit à petit du modèle occidental en direction de l' Océan. Une telle variation spatiale de l'instabilité est aussi observée en France méridionale, où certaines pluies sont concentrées, au hasard des jours, sur tel ou tel petit bassin orographique (ALEXANDRE, 1996, GENIN, 1997).

\section{4 - SYNTHÈSE}

L'on ne peut manquer de rapprocher l'uniformité spatiale de la distribution quotidienne des pluies à l'ouest de celle du peuplement botanique. A l'est, c'est au contraire l'hétérogénéité de la flore qui est à comparer avec celle de la distribution spatiale des pluies. Dans les deux cas, les modalités de variabilité spatiale de la pluie et de la flore sont donc analogues. Ce rapprochement peut sembler curieux, car il serait logique que la composition de la flore dépende plutôt des quantités absolues d'eau reçues que de la manière dont la pluie se distribue dans l'espace !

En fait, cette analogie exprime sans doute une coïncidence. L'atlantisation de la distribution spatiale des précipitations quotidiennes se conjugue en effet avec une hausse rapide de leur montant. L'uniformisation de la flore en direction de l'océan peut alors traduire le simple franchissement d'un seuil de satisfaction hydrique (ALEXANDRE et al., 1996). Au-dessus de ce seuil, les plantes les plus exigentes en eau seraient présentes: la composition de la flore échapperait désormais à l'influence des variations udométriques. La mise en place de la flore atlantique étant complète à la retombée orientale des Serras d'Alvão-Falperra (fig. 2 et 3), c'est là que se localiserait le seuil hydrique. Les précipitations correspondantes avoisineraient $1400 \mathrm{~mm}$ annuels, selon Daveau, 1977 (fig. 1, Minas de Jales), davantage qu'en France, du fait d'une évaporation plus forte au Portugal, qui ne s'atténue vers l'ouest que correlativement avec l'augmentation des pluies. En-dessous de ce seuil, les espèces atlantiques disparaissent vers l'Espagne, progressivement d'abord, sur le plateau du Freixo, puis rapidement à l' est du Tua, la végétation devenant très sensible au dessèchement du 
climat. Le relâchement de la concurrence des espèces atlantiques, vaincues par la sécheresse, permet l'épanouissement de la flore continentale xérique (fig. 3).

L'analyse pollinique confirme l'uniformité botanique du domaine floristique océanique durant la seconde moitié de l'Holocène, où se limitent nos sondages, ce qui exclut pour la situation présente une origine purement anthropique (RUIZ ZAPATA et al.,1993 et 1994).

La consultation des données moyennes mensuelles montre que le régime saisonnier de ce domaine océanique demeure quant à lui tout à fait méditerranéen... surtout à l'approche de l'océan ! Le franchissement du seuil de satisfaction hydrique de la végétation est donc ici tout à fait indépendant de la limite de la zone méditerranénne. Ce franchissement indique seulement une variante biologiquement adoucie du climat méditerranéen, originalité souvent notée du NW de la péninsule ibérique (MOUNIER, 1979, ALCOFORADO et al., 1993)! Vers l'est, la sécheresse s'accompagne d'une continentalisation du régime saisonnier des pluies, dont les maxima tendent à se déplacer vers le début et la fin de la saison chaude (fig. 1, Figueira de Castelo Rodrigo), comme cela s'observe en Afrique septentrionale intérieure (SELTZER, 1946). Cette analogie avec l'Afrique est le résultat de dynamiques saisonnières qui rappellent celles de la zone aride, sauf qu'elles dépendent beaucoup plus ici du dispositif orographique.

\section{CONCLUSION}

Les informations fournies par l'analyse conjointe de la végétation, des pluies et de la circulation atmosphérique quotidienne, enrichissent la géographie climatique portugaise élaborée à partir des valeurs moyennes.

La synthèse de ces informations permet de préciser les lieux de transition majeure du climat et de la végétation, et de déterminer la nature du lien qui les unit. L'existence d'un seuil de satisfaction hydrique de la végétation, placé à la retombée orientale des Serras d'Alvão et Falperra, semble y tenir la première place. A l'ouest de cette limite, la flore atlantique est uniforme; à l'est du rio Tua, elle disparaît rapidement au profit d'espèces plus xérophiles. La vallée encaissée de ce fleuve accuse localement le contraste entre flores occidentale et orientale, accident topoclimatique distinct du seuil hydrique indiqué par la mise en place complète de la flore atlantique.

L'examen du climat au quotidien renouvelle les termes d'une classique discussion (MOUNIER, 1979) sur le caractère méditerranéen ou atlantique du climat du NW de l'Ibérie, qui ne renie à dire vrai ni l'un ni l'autre de ces caractères, mais les reflète tous deux, par le jeu croisé de deux dynamiques planétaires. La première étend à l'ensemble du Portugal la sécheresse relative de l'été méditerranéen. La seconde confère aux pluies océanes de la façade maritime l'abondance et la régularité spatiale que ne connaissent pas les bassins abrités, plus aléatoirement arrosés. L'uniformisation spatiale de la distribution des pluies à l'ouest est concomittante du dépassement du seuil de satisfaction hydrique de la végétation.

Une telle dualité existe aussi plus au sud, au Maroc atlantique, où les perturbations de type norvégien parviennent toutefois essoufflées. Au nord, les deux dynamiques ne se recouvrent plus, la France méditerranéenne, par exemple, n'ayant rien d'atlantique, et la France atlantique étant bien peu méditerranéenne...

\section{BIBLIOGRAPHIE}

Albuquerque, J. De Pina Manique e (1954) - Carta Ecológica de Portugal. Dir. Geral dos Serviços Agrícolas, Lisboa. 
Alcoforado, M. J.; M. F. Alegria; A. Ramos Pereira; C. Sirgado (1993) - Domínios bioclimáticos em Portugal, definidos por comparação dos índices de Gaussen e de Emberger. Linha de Acção de Geografia Física, Rel. n 33, Centro de Estudos Geográficos, reédit., Lisboa.

AlEXANDRE, F. (1996) - Entre midis méditerranéen et atlantique, une transition phytoclimatique du Languedoc à l'Aquitaine. Trav. du Labo. de Géogr. Phys., Univ. Paris 7 - Denis Diderot, 24: 1$-175$.

AleXandre, F.; A. Genin; M. LeCOMPTE (1996) - Seuils hydroclimatiques et limites méditerranéennes. Ann. de Géogr., à paraître.

CORREIA, A.I.D. (1994) - Fitoclimatologia dinâmica, um estudo no norte de Portugal. Tese de Doutoramento em Biologia (Ecologia e Biossistemática), F.C.U.L., Lisboa.

Daveau, S. et al. (1977) - Répartition et rythme des précipitations au Portugal. Memórias do Centro de Estudos Geográficos, no 3, C.E.G., Lisboa.

DELANNOY, H.; M. LECOMPTE (1975) - Etude des régimes thermiques en liaison avec les situations météorologiques dans le Moyen-Atlas (Maroc). Bull. Assoc. Géographes Français, 422-423: 83-94.

DELANNOY, H.; M. LECOMPTE (1980) - Utilisation de l'analyse factorielle des correspondances pour l'étude des précipitations quotidiennes: un exemple au Maroc. Méditerranée, 4: 29-36.

DElanNOY, H.; M. LeCOMPTE; F. RoMANE (1979) - Géographie des températures quotidiennes dans le Moyen-Atlas et ses bordures. Analyse mulltivariée et situations atmosphériques. L'Espace Géographique, 1: 25-40.

DeRrAZ, KH. (1993) - Transition du climat méditerranéen au climat aride dans le Moyen-Atlas oriental marocain: étude phytoclimatique. Thèse de Doctorat, Université de Paris VII, Paris.

DuPONT, P. (1962) - La flore atlantique européenne: introduction à l'étude du secteur ibéro-atlantique, Toulouse.

El Alami, R. (1993) - Phytoclimatologie de la Montagne Marrocaine: le Rif Central. Thèse de Doctorat, Université de Paris VII, Paris.

EMBERGER, L. (1930) - La végétation de la Région Mediterranéenne. Essai d'une classification des groupements végétaux. Rev. Gen. Bot. 42: 641-662 et 705-721.

GAuthier, B.; M. Godron; P. HiernauX; J. LePART (1977) - Un type complémentaire de profil écologique: le profil écologique "indicé". Journal. Canad. Bot., 55 (23): 2859-2865.

GENIN, A. (1997) - Les contacts entre domaines phytoclimatiques: exemple de la bordure cévenole. Trav. du Lab. de Géogr. Phys., Univ. Paris 7 - Denis Diderot, 25: 1-185.

Godron, M. (1967) - Les groupes écologiques imbriqués “en écailles”. Ecol. Plant. 2: 217-226.

Godron, M. (1971) - Essai sur une approche probabiliste de l' écologie des végétaux. Thèse de doctorat d' Etat, Université des Sciences et Techniques du Languedoc, Montpellier.

Guinochet, M. (1973) - Phytosociologie. Masson, Paris.

Humboldt, A.vON (1807) - Essai sur la géographie des plantes. Schoell, Paris.

Lebreton, J. D.; D. Chessel; R. Prodron; N. YocCOZ (1988a) - L'analyse des relations espèces-milieu par l'analyse canonique des correspondances. I. Variables de milieu quantitatives. Acta Ecologica (Ecol. Gener.), 9 (1): 53-67.

Lebreton, J.D.; D. Chessel; M. Richardot-Coulet; N. YocCOZ (1988b) - L'analyse des relations espèces-milieu par l'analyse canonique des correspondances. II. Variables de milieu qualitatives. Acta Ecologica (Ecol. Gener.), 9 (2): 137-151.

LECOMPTE, M. (1973) - Analyse des rapports climat-végétation par une methode d'échantillonnage continu. Bull. Soc. Sc. Nat. et Phys. Maroc, 53 (1-2): 37-61.

LECOMPTE, M. (1981) - Deux approches de la relation climat-végétation. in Eaux et Climats, Mélanges Géographiques offerts en hommage à Ch.P. Péguy, E. R. 30, Grenoble: 303-313.

LECOMPTE, M. (1986) - Biogéographie de la montagne marocaine. Le Moyen-Atlas central. Mémoires et Documents de Géographie, Ed. CNRS, Paris.

LECOMPTE, M. (1987) - Essai de phytoclimatologie dynamique dans le Moyen-Atlas marocain. Acta Ecologica (Ecol. Gener.), 8 (4): 523-535.

LECOMPTE, M. (1988) - La phytoclimatologie dynamique, une approche différente du climat. L'Espace Géographique, 2: 109-121.

LECOMPTE, M. (1989) - La question des limites climatiques à travers la climatologie et la phytoclimatologie dynamiques. La Météorologie, $\mathrm{VII}^{\circ}$ sér., 29: 3-8.

LECOMPTE, M.; F. ALEXANDRE (1996) - Discontinu et continu de la végétation et du milieu - de la théorie des étagements en altitude à la phytoclimatologie dynamique. L'Espace Géographique, 3: 261-272. 
MOUNIER, J. (1979) - Les climats océaniques des régions atlantiques de l' Espagne et du Portugal. Contribution synoptique à la géographie des climats humides des latitudes moyennes. Université de Rennes III.

RIVAS-MARTINEZ, S. (1982) - Étages bioclimatiques, secteurs chorologiques et séries de végétation de 1' Espagne méditerranéenne. Ecol. Medit., 8 (1-2): 317-322.

RonCHAIL, J.; M. LECOMPTE (1981) - Essai de climatologie dynamique dans les Alpes françaises. $L a$ Météorologie, $\mathrm{VI}^{\circ}$ sér., 26: 57-91.

Ruiz Zapata, B.; A. I. D. Correia; S. Daveau; M. LeCompte (1993) - Datos preliminares sobre la evolución de la vegetación en la Sierras del Noroeste de Portugal durante el Holoceno. Actas $3^{a}$ Reunião do Quaternário Ibérico, Coimbra: 97-104.

Ruiz ZapatA, B; A. I. D. CorreiA; S. DAVEAU; M. LeCompte, (1994) - Dinámica de la vegetación durante los últimos 3000 años en la Sierra de Peneda (noroeste de Portugal) en base al análisis polínico. Trabajos de Palinología Básica e Aplicada, Actas X Simposio de Palinología (A.P.L.E.), Valencia: 263-275.

Seltzer, P. (1946) - Le climat de l'Algérie. Inst. de Phys. du Globe de l'Algérie, Alger.

TER BRAAK, C. J. F. (1986) - Canonical Correspondance Analysis: a new eigenvector technic for multivariate Direct Gradient Analysis. Ecology, 7 (5): 1167-1179.

WHITTAKER, R.H. (1967) - Gradient analysis of vegetation. Biol.Rev.,42: 207-264. 